\title{
Enhanced Analysis of WCDMA Networks with Repeaters Deployment
}

\author{
Mario Garcia-Lozano, Luis Alonso, Member, IEEE, Fernando Casadevall, Member, IEEE, \\ Silvia Ruiz, Member, IEEE, and Luis M. Correia, Senior Member, IEEE
}

\begin{abstract}
This paper addresses the analysis of WCDMA systems with repeaters deployment. A generic and compact expression for up- and downlinks evaluation has been mathematically derived so that transmission powers and other radio resource management parameters can be calculated without simplifications. In particular, the real different path delays, taking into account the repeaters presence and the finite nature of the time window of Rake receivers are considered. This allows an enhanced analysis with respect to classical approaches from a system level viewpoint. Furthermore, higher reliable and accurate predictions on network performance can be obtained, which can be remarkably useful for network planning and management. By using these expressions, relevant network parameters have been evaluated and compared with the ones obtained using the classical approximations. The differences in the obtained metrics are highlighted, putting in evidence the improvement provided by the proposed analysis.
\end{abstract}

Index Terms - Network planning, repeaters, WCDMA.

\section{INTRODUCTION}

M OBILE communications systems often work under high heterogeneous conditions, in both time and space domains. Optimizing the design of radio access networks is a technical and economical challenge for operators. In this sense, active repeaters (non-regenerative) are of special interest when considering the coverage of a mobile communications system in a certain set of situations, which include filling in coverage holes in valleys, tunnels, buildings or extending the service area beyond cells boundaries. Despite their use in $2 \mathrm{G}$ (second generation) networks, repeaters are expected to play even a major role in planning WCDMA (Wideband Code Division Multiple Access) systems. Some of the reasons are:

- Since adjacent cells share the same power, repeaters are a cost-effective option to reduce inter-cell interference, particularly in environments with hotspots. If properly adjusted, they can reduce the coupling loss between the base station (BS) and mobile terminals (MT) that are close to the repeater. Therefore, in the uplink (UL),

Manuscript received February 14, 2006; revised October 6, 2006; accepted November 21, 2006. The associate editor coordinating the review of this paper and approving it for publication was K. B. Lee. This work was supported by the European Network of Excellence NEWCOM (Contract number 507325) and by the Spanish Research Council CICYT+FEDER through the projects TEC2005-07326-C02-01/TCM, TIC2003-08609, and TEC2006-09109.

M. Garcia-Lozano, L. Alonso, F. Casadevall, and S. Ruiz are with the Universitat Politècnica de Catalunya (UPC), Av. Canal Olímpic s/n., 08860 Barcelona, Spain (e-mail: \{mariogarcia, luisg, ferranc, silvia\}@tsc.upc.edu).

L. M. Correia is with the Instituto Superior Técnico (IST), Technical University of Lisbon, Av. Rovisco Pais, IT/DEEC Torre Norte, 1049-001 Lisboa, Portugal (e-mail: luis.correia@1x.it.pt).

Digital Object Identifier 10.1109/TWC.2007.06114.
MTs use a lower output power and the interference in neighbouring cells is reduced. The work in [1] studies the use of repeaters in WCDMA systems with hotspots and reveals this fact among others. Subsequently, the BS that is connected to a repeater will be denoted as its donor BS.

- Radio over fibre and optical wireless are mature enough technologies to allow a dense deployment of repeaters, especially in urban environments. Paper [2] describes two radio over fibre systems, for GSM and UMTS networks. In the first case, the article also describes a real and complete system as well as results obtained in fieldtests, which were designed to improve coverage inside a building.

- Indeed the use of higher frequencies in WCDMA systems will imply higher propagation losses. Repeaters can be key devices to guarantee indoor coverage at a low cost.

- During the last years, repeaters have improved in terms of operation and maintenance capabilities.

Unfortunately, since repeaters are not noiseless devices, they modify the interference and thermal noise patterns of the donor BS receiver (RX). This implies an effect in both the coverage and capacity of the cell. Since the noise floor of the donor is raised, its effective coverage area is shrunk. However, this will be clearly compensated by the new areas, covered by the repeaters. On the other hand, the maximum allowed load factor is more likely to be reached, and so another effect is the reduction of the admission region in the donor BS [3].

In a mobile communications system with repeaters, signal contributions from the donor BS and from repeaters arrive through different paths, and those ones passing through a repeater are usually 'longer' (both in spatial and time domains). This is because of the delay introduced by the link between the repeater and the donor BS, and the internal delay of the repeater itself. In fact, the link can be established via various transmission media, such as fiber optic or copper, which show some advantages compared to radio, but also imply lower propagation velocities. On the other hand, the Rake RX performs a delay profile characterization [4] and just those components within a certain time window are constructively combined. Therefore, with the introduction of repeaters, the system analysis is significantly modified and must be revised.

Although much previous research efforts have been focused on the analysis of CDMA-based systems ([5]-[7]), only to cite a few), not many studies in the literature analyze the effect of repeaters on the capacity and feasibility conditions 
for a CDMA mobile communications system. In [8], the authors study repeaters as coverage extenders and how they modify interference in highways environments. Similarly, [3] and [9] deal with coverage, but also investigate variations in capacity due to the noise rise that appears in the donor BS when repeaters are installed. In order to minimize this effect, [10] proposes an automatic on-off switching repeater. Finally an analytic scheme for estimating the received signal power required at the BS front-end in an environment with fibreoptic repeaters is found in [11]. On the other hand, published studies always consider one of following assumptions:

1) $\mathrm{RXs}$ receive power just through the radio-link with the donor $\mathrm{BS}$ or through one of its repeaters. Other contributions are not considered in any way [10], [11].

2) All the contributions are always perfectly combined in the MT. Ideal maximum ratio combining (MRC) is applied [1], [3], [8].

The work in [9] evaluates the two situations independently, and also another one that is in the middle of them.

However, in comparison to classical approaches, in real systems the Rake RX has to perform a delay profile measurement to designate the time offsets of the strongest signal components. Only those paths within a certain time window are constructively combined, the others not being considered and causing a certain level of 'self-interference'. UMTS (Universal Mobile Telecommunications System) BSs and MTs can usually handle a $20 \mu$ s time delay between two paths [12], and this value must be considered when repeaters are deployed, since MTs can incur into high levels of multi-path [13].

The novelty of this paper is a generic and complete analysis of a WCDMA system with repeaters from a system level viewpoint. Compact expressions for the UL and downlinks (DL) are derived, so that transmission powers and other radio resource management (RRM) parameters can be calculated without any of the previously mentioned simplifications. This allows an enhanced prediction of the network performance when studying WCDMA environments with repeaters deployment. Moreover, a comparison with the results obtained using classical approaches has also been done. Different relevant parameters that characterize networks performance have been evaluated and compared. The differences with respect to simplified analysis are shown to be remarkable, stressing the improvement provided by the proposed analysis.

The paper is organized in 5 differentiated sections. After this introduction, the model is explained, as well as the notation subsequently used, in Section II. Next, in Section III the mathematical approach is developed along two subsections, the first one being devoted to the UL and the second one to the DL, some comments on final equations being also given. Section IV contains numerical results and, finally, Section V closes the paper with the conclusions derived from the work.

\section{System Model}

Let us consider a mobile communications systems consisting of any number $N_{B S}$ of BSs, each one of them connected to any number of repeaters, $N_{\text {Rep }}(j)$ being the number of repeaters connected to $\mathrm{BS} j$. The system layout can be completely generic, with no restriction on the spatial configuration

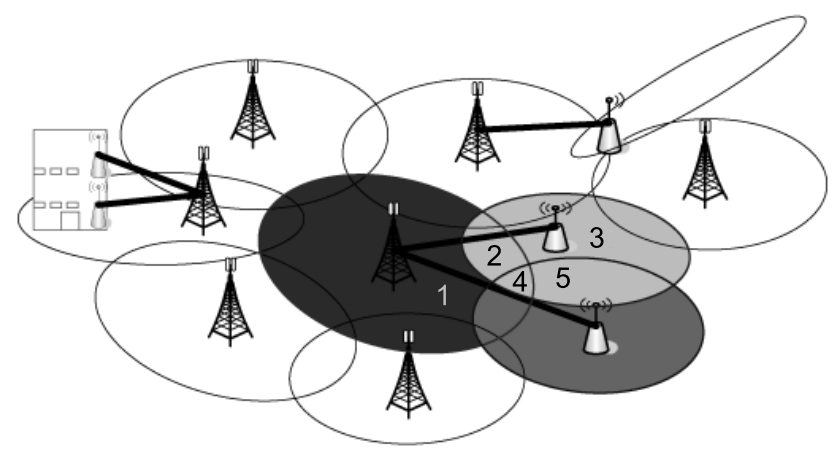

Fig. 1. Different reception situations in an environment with repeaters.

and deployment of both the BS and the repeaters, and the links between BS and repeaters can also be of any nature. It is assumed that there are $N_{M T}$ MTs in the network, spread all around the area under study without any restriction; there are no restrictions on the service the MTs can use, either. A power control algorithm is present, being composed of the so-called inner and outer loops. The former aims at adjusting the transmitted powers, so that a certain signal to interference plus noise ratio (SINR) target before de-spreading for a user $k$ is reached; this ratio is denoted as $\gamma\left(k, s_{k}\right), s_{k}$ being the BS that serves $k$. The latter intends to keep the quality of communications at a desired level in terms of block error rate (BLER) depending on higher layers' requirements. The expression for $\gamma\left(k, s_{k}\right)$ is straightforward in the case of a generic WCDMA system without repeaters deployment [4], but, with the introduction of repeaters, the analysis has to be modified. The different cases to be considered are shown by means of an example in Fig. 1. Indeed, based on the position of an MT with respect to the network layout, the signal could be received in different ways (numbered in the figure):

1) Directly from the donor BS.

2) Simultaneously from the donor BS and one repeater.

3) Just from one repeater.

4) From the donor BS and several repeaters.

5) From several repeaters.

6) All the previous ones in a soft-handover situation with other BSs in the system or repeaters connected to other BSs.

For example, type 2 areas are defined as the zones in which the difference of propagation times of both received paths (in this case from one BS and one of its repeaters) is smaller than the Rake time window. Otherwise, one of the paths would generate a level of self-interference, which is nonexistent in a deployment without repeaters. In this sense, the position of the MT with respect to each BS or repeater, the internal delay introduced by the repeater, and the transmission media in the link between the donor and the repeater are of key importance.

Lets us define $\Gamma(k, j)$ as the set of mean propagation losses of the different paths between MT $k$ and BS $j$, of dimension $N_{P}$, that is:

$$
\Gamma(k, j) \equiv\left\{L_{1}(k, j), L_{2}(k, j), \ldots, L_{N_{P}}(k, j)\right\}
$$

Where each element $L_{i}(k, j)$ of the set represents the absolute propagation loss of the $i$-th transmission path from 
MT $k$ to BS $j$ :

$$
\begin{aligned}
\Gamma(k, j)= & \left\{L_{M T-B S}(k, j), \frac{L_{M T-R e p}\left[k, r_{1}(j)\right] \cdot L_{R e p-B S}\left[r_{1}(j), j\right]}{G_{R e p}\left[r_{1}(j)\right]},\right. \\
& \left.\ldots, \frac{L_{M T-R e p}\left[k, r_{N_{R e p}(j)}(j)\right] \cdot L_{R e p-B S}\left[r_{N_{R e p}(j)}(j), j\right]}{G_{R e p}\left[r_{N_{R e p}(j)}(j)\right]}\right\}
\end{aligned}
$$

Where:

- $L_{M T-B S}(k, j)$ is the radio propagation loss between $k$ and $j$.

- $L_{M T-R e p}(k, r)$ is the radio propagation loss between $k$ and a repeater $r$.

- $L_{R e p-B S}(r, j)$ is the loss between repeater $r$ and $j, r_{i}(j)$ being the $i$-th repeater connected to $j$.

- $G_{R e p}(r)$ is the power gain provided by repeater $r$, being properly selected in order to avoid the saturation of the repeater amplifier.

The subset $\Psi(k, j) \subset \Gamma(k, j)$ is defined as the one containing the paths that are constructively combined by the Rake RX, that is, those within the Rake observing time window limits.

\section{SIR ANALYSIS}

The objective of this section is to derive a generic expression so that transmission powers can be realistically found in order to achieve the system requirements. This is done for each link (UL and DL), valid for all types of system layouts, irrespectively of the position of the MTs with respect to the BS or the repeaters. The following subsections are devoted separately to each link.

\section{A. Uplink}

Taking the system model and definitions into account, the SINR for MT $k$ in UL before de-spreading $\gamma^{U L}\left(k, s_{k}\right)$, can be written as:

$$
\begin{aligned}
& \gamma^{U L}\left(k, s_{k}\right) \equiv \\
& \equiv \frac{\sum_{i \in \Psi\left(k, s_{k}\right)} P_{T X}^{U L} / L_{i}\left(k, s_{k}\right)}{I^{U L}\left(k, s_{k}\right)+\tilde{P}_{R X}\left(k, s_{k}\right)+n_{B S}\left(s_{k}\right)+n_{R X, R e p}\left(s_{k}\right)}
\end{aligned}
$$

Where:

- $P_{T X}^{U L}(k)$ : The transmission power used by $k$.

- $I^{U L}\left(k, s_{k}\right)$ : The UL multi-user interference power received by the connection between $k$ and $s_{k}$, for both inter- and intra-cells.

- $\tilde{P}_{R X}\left(k, s_{k}\right)$ The self-interference power of $k$ at $s_{k}$, being generated by signal replicas from $k$ that arrive with such a delay that fall out of the Rake window.

- $n_{B S}(j)$ : The thermal noise power produced by the BS RX.

- $n_{R X, R e p}(j)$ : The received thermal noise power produced by all repeaters connected to BS $j$ :

$$
n_{R X, \operatorname{Rep}}(j)=\sum_{i=1}^{N_{R e p}(j)} \frac{n_{R e p}\left[r_{i}(j)\right] \cdot G_{R e p}\left(r_{i}\right)}{L_{R e p-B S}\left[r_{i}(j), j\right]}
$$

Note that the last two terms in the denominator are constant and can be grouped in a single thermal noise term, which is called $n\left(s_{k}\right)$.

The numerator of (3) is the constructive addition of the signal's replicas that can be combined in the Rake RX. On the other hand, the term $I^{U L}\left(k, s_{k}\right)$ takes into account all the possible paths of interfering signals for all users in the system. Moreover, the self-interference level of $k$ at $s_{k}$ can be expressed as:

$$
\tilde{P}_{R X}\left(k, s_{k}\right) \equiv \sum_{\substack{i \in \Gamma\left(k, s_{k}\right) \\ i \notin \Psi\left(k, s_{k}\right)}} P_{T X}^{U L}(k) / L_{i}\left(k, s_{k}\right)
$$

Consequently, using the previous expressions, (3) can be rewritten as:

$$
\gamma^{U L}\left(k, s_{k}\right)=\frac{\sum_{i \in \Psi\left(k, s_{k}\right)} \frac{P_{T X}^{U L}(k)}{L_{i}\left(k, s_{k}\right)}}{\sum_{\substack{m=1 \\ m \neq k}}^{N_{M T} T\left(k, s_{k}\right)} \frac{P_{T X}^{U L}(m)}{L_{i}\left(m, s_{k}\right)}+\sum_{\substack{i \in \Gamma\left(k, s_{k}\right) \\ j \notin \Psi\left(k, s_{k}\right)}} \frac{P_{T X}^{U L}(k)}{L_{i}\left(k, s_{k}\right)}+n\left(s_{k}\right)}
$$

Some papers dealing with repeaters (e.g., [8]) tend to make use of the quotient $I_{\text {other }} / I_{\text {self }}$, the ratio of other cell to own cell interferences. The use of this factor is justified in many cases to obtain tendencies and first ideas about the behaviour of a network. However, in this case, for both UL and DL, this relationship has been avoided because the proposal aims at solving the system realistically.

From (6), it can be seen that the useful received power at $s_{k}$ from $k$ (numerator) can be expressed as the product between the transmitted power and a certain term that only depends on propagation conditions. Then, it is possible to see the inverse of this factor as an 'effective' equivalent loss. Looking at the numerator, this term is calculated as the parallel (using an electrical equivalency) of the loss of the paths inside the time window of the Rake RX. It can also be observed that this idea can be extrapolated to other terms of (6). Indeed, the self-interference can also be written as the transmitted power divided by another effective loss, which is evaluated as the parallel of those paths not being constructively combined at the Rake RX. Finally, interference from any other MT $m$ in the system is its transmitted power divided by the parallel of all paths between itself and the BS. Then, the following notation definitions are taken:

$$
\begin{aligned}
L_{e f \Gamma}(k, j) & =\left(\sum_{i \in \Gamma\left(k, s_{k}\right)} \frac{1}{L_{i}(k, j)}\right)^{-1} \\
L_{e f \Psi}(k, j) & =\left(\sum_{i \in \Psi\left(k, s_{k}\right)} \frac{1}{L_{i}(k, j)}\right)^{-1} \\
L_{e f \Gamma-\Psi}(k, j) & =\left(\sum_{\substack{i \in \Gamma\left(k, s_{k}\right) \\
i \notin \Psi\left(k, s_{k}\right)}} \frac{1}{L_{i}(k, j)}\right)^{-1}
\end{aligned}
$$


which correspond, respectively, to the effective path loss of all possible propagation paths between $k$ and $j$, the effective path loss of only the paths constructively combined within the Rake RX, and the effective path loss considering the paths with such a delay that they appear outside the Rake time window. With this notation, some terms in (6) can be simplified as:

$$
\begin{gathered}
P_{T X}^{U L}(k) \sum_{i \in \Psi\left(k, s_{k}\right)} \frac{1}{L_{i}\left(k, s_{k}\right)}=\frac{P_{T X}^{U L}(k)}{L_{e f \Psi}\left(k, s_{k}\right)} \\
\tilde{P}_{R X}\left(k, s_{k}\right)=P_{T X}^{U L}(k) \sum_{\substack{i \notin \Psi\left(k, s_{k}\right) \\
i \in \Gamma\left(k, s_{k}\right)}} \frac{1}{L_{i}\left(k, s_{k}\right)}=\frac{P_{T X}^{U L}(k)}{L_{e f \Gamma-\Psi}\left(k, s_{k}\right)}
\end{gathered}
$$

$$
P_{T X}^{U L}(m) \sum_{i \in \Gamma\left(k, s_{k}\right)} \frac{1}{L_{i}\left(m, s_{k}\right)}=\frac{P_{T X}^{U L}(m)}{L_{e f \Gamma}\left(m, s_{k}\right)}, \forall m \mid m \neq k
$$

Using all these notations in (6), we can write a more compact expression for $\gamma^{U L}\left(k, s_{k}\right)$ :

$$
\begin{aligned}
\gamma^{U L}\left(k, s_{k}\right) & =\frac{\frac{P_{T X}^{U L}(k)}{L_{e f \Psi}\left(k, s_{k}\right)}}{\sum_{i=1}^{N_{M T} T} \frac{P_{T X}^{U L}(i)}{L_{e f \Gamma}\left(i, s_{k}\right)}-\frac{P_{T X}^{U L}(k)}{L_{e f \Psi}\left(k, s_{k}\right)}+n\left(s_{k}\right)} \\
& =\frac{\frac{P_{T X}^{U L}(k)}{L_{e f \Psi}\left(k, s_{k}\right)}}{P_{R X, t o t}^{U L}\left(s_{k}\right)-\frac{P_{T X}^{U L}(k)}{L_{e f \Psi}\left(k, s_{k}\right)}}
\end{aligned}
$$

where $P_{R X, t o t}^{U L}(j)$ is the total power received at $j$, including all the signal and thermal noise terms.

$$
P_{R X, t o t}^{U L}(j)=\sum_{i=1}^{N_{M T}} P_{T X}^{U L}(i) / L_{e f \Gamma}(i, j)+n(j)
$$

Next, in order to further simplify (13), let us define $\phi^{U L}$ as the SSIR (Signal to Signal-plus-Interference-plus-Noise Ratio) measured in the UL:

$\phi^{U L}\left(k, s_{k}\right) \equiv \frac{\gamma^{U L}\left(k, s_{k}\right)}{1+\gamma^{U L}\left(k, s_{k}\right)}=\frac{P_{T X}^{U L}(k)}{P_{R X, t o t}^{U L}\left(s_{k}\right) \cdot L_{e f \Psi}\left(k, s_{k}\right)}$

Thus, we finally obtain a compact and general expression that allows calculating the power that an MT must transmit to reach a certain SIR target:

$$
P_{T X}^{U L}(k)=\phi^{U L}\left(k, s_{k}\right) \cdot P_{R X, t o t}^{U L}\left(s_{k}\right) \cdot L_{e f \Psi}\left(k, s_{k}\right)
$$

Notice that the required transmission power depends on the total amount of power received at the server BS and the effective path loss (parallel) of those paths within the Rake time window. Obviously, it also depends on the SIR target. Observe that the total effective loss, that is, considering all transmission paths, is also being considered within the total received power term.

It is obvious that the greater the number of repeaters, the higher the dimension of $\Gamma$, but also, potentially, the dimension of $\Psi$. This would imply a decrease in $L_{e f \Gamma}\left(k, s_{k}\right)$ and consequently an interference increase; on the other hand, it would also imply a decrease in $L_{e f \Psi}\left(k, s_{k}\right)$ and therefore a reduction in the required power, see (8). Then, the global increase or reduction of $P_{T X}^{U L}(k)$ depends on the relative position of the BS and repeaters with respect to the users, not strictly only in a geographical viewpoint but also in the radiofrequency propagation domain. From (16), it is clear that the transmitted powers can be calculated by solving the $N_{M T}$ th-order linear equations system formed by the application of this expression for each one of the MTs in the system. In general, $N_{M T}$ is high, therefore, this type of 'microscopic' approach requires a lot of computational power, especially if it has to be solved on a frame-by-frame basis. Nevertheless, a second relationship between $P_{R X, t o t}^{U L}(j)$ and the individual UL transmitted powers was established in (14), so that the complexity of the problem can be turned into a 'macroscopic' approach, while remarkably reducing the computational cost. Indeed, we can write that:

$$
\begin{aligned}
& P_{R X, t o t}^{U L}(j)= \\
& \quad=\sum_{i=1}^{N_{M T}}\left[\frac{L_{e f \Psi}\left(i, s_{i}\right)}{L_{e f \Gamma}(i, j)} \cdot \phi^{U L}\left(i, s_{i}\right) \cdot P_{R X, t o t}^{U L}\left(s_{i}\right)\right]+n(j)
\end{aligned}
$$

where (16) has been used to substitute the numerator of the first term of (14). As a consequence, the dimension of the equations system can be reduced to $N_{B S}$, in the same way as it is usually done in WCDMA systems without repeaters deployment [14], [15]. Since $N_{B S}$ is far smaller than $N_{M T}$, the analysis is considerably reduced in terms of computational cost. The final linear equations system can be expressed in matrix notation as follows:

$$
\boldsymbol{\Omega}_{\mathrm{UL}} \cdot \Pi_{\mathrm{RX}, \text { tot }}^{\mathrm{UL}}=\mathrm{N}_{\mathrm{UL}}
$$

where $\Pi_{\mathbf{R X} \text {,tot }}^{\mathrm{UL}}$ is a vector containing the unknowns, that is, the total received power at each BS:

$$
\Pi_{\mathbf{R X}, \text { tot }}^{\mathrm{UL}}(j)=P_{R X, t o t}^{U L}(j)
$$

$\mathrm{N}_{\mathrm{UL}}$ is a vector with the total thermal noise power (received from repeaters and the BS itself).

$$
\mathrm{N}_{\mathbf{U L}}(j)=n(j)
$$

and $\boldsymbol{\Omega}_{\mathrm{UL}}$ representing a $N_{B S} \times N_{B S}$ matrix with individual elements calculated as follows, $\delta_{j, i}$ being the Kronecker delta:

$$
\boldsymbol{\Omega}_{\mathbf{U L}}(j, i)=\delta_{j, i}-\sum_{\substack{m=1 \\ m \in i}}^{N_{M T}} \frac{L_{e f \Psi}(m, i)}{L_{e f \Gamma}(m, j)} \cdot \phi(m, i)
$$

The summation is calculated over the MTs connected to BS $i$, which is indicated by ' $m \in i$ ', $m$ being the summation index.

Note that the number of equations does not depend on the number of installed repeaters. Therefore, the computational cost to analyze the network with repeaters deployment is independent of their number, and we can afford using as much repeaters as needed without adding significant complexity 
to the network planning. Once the total received power has been calculated, the individual MT transmitted power can be calculated by using (16).

MTs in soft handover can be also considered with minor changes in the formulation, which is an additional usefulness. In order to take these users into account, the different BSs to which the MT is connected (subsequently called active set) should be evaluated independently and, a posteriori, the connection that requires less power from the user should be selected. From an analysis viewpoint, if MT $k$ has $\varepsilon(k)$ BSs in its active set, it would be characterized as $\varepsilon(k)$ independent MTs, each one connected to a different BS. Obviously, this indirectly implies a higher number of connections in the network and a more hostile scenario in terms of interference, as:

$$
\sum_{i=1}^{N_{M T}} \varepsilon(i)=N_{M T}^{\prime} \geq N_{M T}
$$

In this situation, it is necessary to solve the linear equations system twice. Firstly, a coarse adjust is done, with all virtual connections. Subsequently, a refined one should be done, once the optimum connection is chosen and the others are eliminated. Note that the BS that requires less power from the MT may not be the same as the best BS in the active set, which is selected according to the $E_{c} / I_{0}$ levels measured on the common pilot channel.

\section{B. Downlink}

For DL, the objective of the analysis is to find a generic expression to evaluate the power that a certain BS $j$ has to transmit to a given MT $k, P_{T X}^{D L}(j, k)$, in order to meet all the users' requirements. Similarly to UL, and using the same notation, it is possible to write one single equation to calculate SINR:

$$
\gamma^{D L}(j, k)=\frac{P_{T X}^{D L}(j, k) / L_{e f \Psi}(k, j)}{I_{\text {Inter }}^{D L}(j, k)+I_{\text {Intra }}^{D L}(j, k)+n_{M T}(k)}
$$

where $I_{\text {Inter }}^{D L}(j, k)$ is the inter-cell interference, $I_{\text {Intra }}^{D L}(j, k)$ stands for intra-cell one, and $n_{M T}(k)$ represents the total thermal noise power measured at MT $k$. Note that, in order to simplify notation, the same denomination for effective propagation losses has been used in both links, although these values may not match in FDD systems. Then, $L_{e f \Psi}(k, j)$ stands for the effective path loss from BS $j$ to MT $k$ considering only the paths that will be constructively added in the Rake receiver at $k$.

Note that in WCDMA systems such as UMTS, on the DL, all BSs in the active set are simultaneously transmitting. So if the user is in soft handover, $I_{\text {Inter }}^{D L}(j, k)$ will depend on the specific BS link that is evaluated. Moreover, if $j$ does not belong to the MT's active set, then $P_{T X}^{D L}(j, k)=0$. Therefore, $I_{\text {Inter }}^{D L}(j, k)$ can be written as:

$$
I_{\text {Inter }}^{D L}(j, k) \equiv \sum_{\substack{i=1 \\ i \neq j}}^{N_{B S}} \frac{P_{T X, t o t}^{D L}(i)}{L_{e f \Gamma}(k, i)}
$$

where $P_{T X, t o t}^{D L}(j)$ is the total transmission power of BS $j$ including the power devoted to MTs and control channels.

Concerning, $I_{I n t r a}^{D L}(j, k)$, the interference measured by $k$ at the link with $j$, and caused by the BS itself, it is the summation of powers transmitted by the BS towards the rest of users in the cell plus the power devoted to control channels $c(j)$, measured at $k$. Ideally, the interference should be zero, because of the use of orthogonal codes [4] (OVSF family codes in the UMTS case); however, in a real system a certain loss of orthogonality exists due to multi-path propagation. The effective interference will be then characterized by a fraction of the total power, by means of the so-called orthogonality factor $\rho(j, k) \in[0,1]$, whose value will depend on the type of environment and on the power delay profile of the channel. Note that we define $\rho=1$ as a situation with full loss of orthogonality. It must be pointed out that if $j$ does not belong to the active set of $k$, the intra-cell interference will be zero. Finally, a selfinterference term can also potentially appear in DL, and it is also included as part of the intra-cell interference. Given all these definitions, $I_{\text {Intra }}^{D L}(j, k)$ can be expressed as (25). Note that the summation is calculated over the MTs connected to BS $j$, which is indicated by ' $i \in j$ ', $i$ being the summation index.

$$
\begin{aligned}
& I_{\text {Intra }}^{D L}(j, k) \equiv \\
& \equiv \rho(j, k)\left[\frac{c(j)}{L_{e f \Gamma}(k, j)}+\frac{P_{T X}^{D L}(j, k)}{L_{e f \Gamma-\Psi}(k, j)}+\sum_{\substack{i=1 \\
i \neq k \\
i \in j}}^{N_{M T}} \frac{P_{T X}^{D L}(j, i)}{L_{e f \Gamma}(k, j)}\right] \\
& =\rho(j, k)\left[\frac{P_{T X, t o t}^{D L}(j)}{L_{e f \Gamma}(k, j)}+\frac{P_{T X}^{D L}(j, k)}{L_{e f \Gamma-\Psi}(k, j)}-\frac{P_{T X}^{D L}(j, k)}{L_{e f \Gamma}(k, j)}\right] \\
& =\rho(j, k)\left[\frac{P_{T X, t o t}^{D L}(j)}{L_{e f \Gamma}(k, j)}-\frac{P_{T X}^{D L}(j, k)}{L_{e f \Psi}(k, j)}\right]
\end{aligned}
$$

Note that the total power transmitted by a BS $j, P_{T X, t o t}^{D L}(j)$, is expressed as:

$$
P_{T X, t o t}^{D L}(j) \equiv c(j)+\sum_{\substack{i=1 \\ i \in j}}^{N_{M T}} P_{T X}^{D L}(j, i)
$$

If these expressions are substituted in (23), a new expression for $\gamma^{D L}(j, k)$ can be obtained:

$$
\begin{aligned}
& \gamma^{D L}(j, k)= \\
& =\frac{\frac{P_{T X}^{D L}(j, k)}{L_{e f \Psi}(k, j)}}{\sum_{\substack{i=1 \\
i \neq j}}^{N_{B S}} \frac{P_{T X, t o t}^{D L}(i)}{L_{e f \Gamma}(k, i)}+\rho(j, k)\left[\frac{P_{T X, t o t}^{D L}(j)}{L_{e f \Gamma}(k, j)}-\frac{P_{T X}^{D L}(j, k)}{L_{e f \Psi}(k, j)}\right]+n_{M T}(k)} \\
& =\frac{\frac{P_{T X}^{D L}(j, k)}{L_{e f \Psi}(k, j)}}{P_{R X, t o t}^{D L}(k)-\rho(j, k) \frac{P_{T X}^{D L}(j, k)}{L_{e f \Psi}(k, j)}}
\end{aligned}
$$

The total received power at $\mathrm{k}, P_{R X, t o t}^{D L}(k)$, has been used in the equation, being given by: 
$P_{R X, t o t}^{D L}(k)=\sum_{\substack{i=1 \\ i \neq j}}^{N_{B S}} \frac{P_{T X, t o t}^{D L}(i)}{L_{e f \Gamma}(k, i)}+\rho(j, k) \frac{P_{T X, t o t}^{D L}(j)}{L_{e f \Gamma}(k, j)}+n_{M T}(k)$

Similarly to the UL case, this expression can be manipulated and simplified. However, in this case the SSIR will not be directly used. Instead, a derived term $\phi_{\rho}(j, k)$ containing the orthogonality factor is defined and simplified by using (28):

$$
\phi_{\rho}(j, k) \equiv \frac{\gamma^{D L}(j, k)}{1+\rho(j, k) \cdot \gamma^{D L}}=\frac{P_{T X}^{D L}(j, k)}{L_{e f \Psi}(k, j) \cdot P_{R X, t o t}^{D L}(k)}
$$

Finally, from the previous expression, it is possible to obtain the power that $j$ should transmit to $k$. Note that if $j$ does not appear in the active set of $k, \gamma^{D L}(j, k)$, and thus $P_{T X}(j, k)$, are equal to zero. Then, this power can be formulated as:

$$
P_{T X}^{D L}(j, k)=\phi_{\rho}(j, k) \cdot L_{e f \Psi}(k, j) \cdot P_{R X, t o t}^{D L}(k)
$$

It can be seen that this expression is almost symmetrical to the one obtained for UL, and that the conclusions drawn are applicable. Again, this final equation allows finding the unknowns, $P_{T X}^{D L}(j, k)$, by solving a $N_{M T}$ th-order linear equations system. Again, a dimensionality reduction will be an important issue to attain. Then, we propose a procedure similar to that performed for the UL.

Firstly, the total received power by $k$ can be found directly from the total transmitted power by all BSs, (28). On the other hand, $P_{T X, t o t}^{D L}(j)$ is the sum of the individual transmitted powers to the MTs connected to $j$ plus the power devoted to control channels. Obviously, this sum cannot exceed the maximum power available at the $\mathrm{BS}, P_{T X, \max }^{D L}(j)$. Moreover, note that a user with several BSs in its active set will contribute to all the summations of those BSs as:

$$
P_{T X, t o t}^{D L}(j) \equiv \sum_{\substack{i=1 \\ i \in j}}^{N_{M T}} P_{T X}^{D L}(j, i)+c(j) \leq P_{T X, \max }^{D L}(j)
$$

Given this, if (30) is substituted in (31), we get the expression that will allow reducing the dimension of the problem,

$$
\begin{aligned}
P_{T X, t o t}^{D L}(j) & =\sum_{\substack{m=1 \\
m \in j}}^{N_{M T}} \phi_{\rho}(j, m) \cdot L_{e f \Psi}(j, m) \cdot \\
\cdot & {\left[\sum_{i=1}^{N_{B S}} \frac{P_{T X, t o t}^{D L}(i) \cdot \rho(i, m)}{L_{e f \Gamma}(m, i)}+n_{M T}(m)\right]+c(j) }
\end{aligned}
$$

From this point, the problem can be posed again as a $N_{B S}$ th-order linear equations system,

$$
\Omega_{\mathrm{DL}} \cdot \Pi_{\mathrm{TX}, \text { tot }}^{\mathrm{DL}}=\Phi_{\mathrm{DL}}
$$

where $\Pi_{\mathbf{T X}, \text { tot }}^{\mathrm{DL}}$ is a vector of dimension $N_{B S}$ containing the unknowns:

$$
\boldsymbol{\Pi}_{\mathbf{T X}, \mathbf{t o t}}^{\mathrm{DL}}(j)=P_{T X, t o t}^{D L}(j)
$$

$\Omega_{\mathrm{DL}}$ represents a matrix of dimension $N_{B S} \times N_{B S}$ with individual values calculated as follows:

$$
\boldsymbol{\Omega}_{\mathbf{D L}}(j, i)=\delta_{j, i}-\sum_{\substack{m=1 \\ m \in j}}^{N_{M T}}\left[\frac{L_{e f \Psi}(j, m) \cdot \rho(i, m)}{L_{e f \Gamma}(m, i)} \cdot \phi_{\rho}(j, m)\right]
$$

and $\boldsymbol{\Phi}_{\mathbf{D L}}$ is a vector of dimension $N_{B S}$ with individual elements calculated as follows:

$$
\boldsymbol{\Phi}_{\mathbf{D L}}(j)=c(j)+\sum_{\substack{i=1 \\ i \in j}}^{N_{M T}} \phi_{\rho}(j, i) \cdot L_{e f \Psi}(j, i) \cdot n_{M T}(i)
$$

Thus, after solving (33), the individual transmitted powers can be calculated for each MT by applying (30). Since the resolution of the linear equations system can give impossible solutions not fulfilling the inequality in (31), before calculating the individual powers the terms in $\Pi_{\mathbf{T X}, \text { tot }}^{\mathrm{DL}}$ should be limited to the maximum available power at each BS.

In this way, a complete description and analysis of WCDMA networks is given when repeaters are deployed. We want to remark that all this analysis does not include any type of approximation regarding the combination of the signals at the Rake RX and all the signal terms present in the system (both useful and interference). Therefore, it allows characterizing the system with the same computational cost as classical approaches do, but considering the inclusion of repeaters in the system deployment and getting a higher accurate solution.

\section{Numerical Results}

In order to evaluate several performance indicators of a WCDMA network, and to quantify the differences obtained when using the proposed analysis with respect to classical approaches (which include some approximations and simplifications), simulations have been run. All the numerical analyses described in the previous section have been embedded in the simulator, along with the assumptions that were described in Section II. Particularly, a system level simulator has been developed based on Monte Carlo experiments, which means that several snapshots of the network are sequentially generated and simulated. Experiments are independent among them, as a consequence MTs are randomly and uniformly scattered around the scenario at the beginning of each sample. The number of MTs in the first set of simulations has been adjusted so that the number of users not reaching the $E_{b} / N_{0}$ required by the power control is $5 \%$ when using the MRC approach. This implies 93 users inside the pilot channel coverage area (details on coverage calculations will be given later on).

The study case scenario consists of a long road or railway like scenario, with one $\mathrm{BS}$ and one repeater that has been used as a coverage extender. The distance between the donor BS and the repeater is $4 \mathrm{~km}$ and the repeater has been installed in the direction of maximum gain of the BS's antenna. The objective is to cover a long portion of the road or railway. The link between them is considered to be an optical fibre with refraction index of 1.48 in the core and a length of $4.5 \mathrm{~km}$. 
TABLE I

Simulation PARAMETERS

\begin{tabular}{|c|c|c|c|}
\hline \multicolumn{2}{|c|}{ Maximum number of BSs in the active set } & \multicolumn{2}{|l|}{3} \\
\hline & odiversity window size & 3 & $\mathrm{~dB}$ \\
\hline \multicolumn{2}{|c|}{ Minimum required common pilot channel $E_{c} / I_{0}$} & -12 & $\mathrm{~dB}$ \\
\hline \multirow[t]{2}{*}{ BS } & Max. transmission power & 43 & $\mathrm{dBm}$ \\
\hline & Noise Figure & 5 & $\mathrm{~dB}$ \\
\hline \multirow[t]{2}{*}{ MT } & Max. transmission power & 21 & $\mathrm{dBm}$ \\
\hline & Noise Figure & 8 & $\mathrm{~dB}$ \\
\hline \multirow[t]{2}{*}{ Repeater } & Max. transmission power & 43 & $\mathrm{dBm}$ \\
\hline & Noise Figure & 5 & $\mathrm{~dB}$ \\
\hline \multicolumn{2}{|r|}{ UL Eb/N0 target } & 2.9 & $\mathrm{~dB}$ \\
\hline \multicolumn{2}{|r|}{$\mathrm{DL}$ Eb/N0 target } & 4.4 & $\mathrm{~dB}$ \\
\hline \multicolumn{2}{|c|}{ Maximum DL power per connection } & 21 & $\mathrm{dBm}$ \\
\hline \multicolumn{2}{|r|}{ Service Bit Rate } & 12.2 & kbps \\
\hline
\end{tabular}

It is considered that the fibre is not installed along a perfect straight line between the BS and the repeater, but rather it can be part of a ring, continue railway path, etc. The gains of the transmitter and receiver in the link and the internal gain of the repeater are adjusted so that there is no amplifier saturation at the repeater. For radio propagation evaluation, the classical COST231-Hata propagation model for suburban areas has been used [16], considering a $2 \mathrm{GHz}$ carrier and radiation patterns from commercial antennas [17]. A summary of the main parameters values is shown in Table I.

Different curves show the behaviour of MTs for different internal delays at the repeater and other active devices in the link between the donor BS and the repeater (from 5 to 11 $\mu \mathrm{s})$, however note that what it is important is the difference between the absolute delays of the paths. The same results can be obtained for smaller internal delays in the repeaters, but for a longer fibre, etc. The curves with caption MRC and SEL stand for the cases in which classical approaches were used. Specifically:

- SEL: RXs receive power just through the radio-link with the BS or through one of the repeaters. Other contributions are not considered in any way. Note that this case is different from our proposal when only one path is recovered: those paths that are not used to recover the signal do generate interference and are taken into account as well.

- MRC: All contributions are always perfectly combined in the MT irrespectively of their different delays. Ideal maximum ratio combining of all paths is applied. Please see Section I for references about SEL and MRC cases.

Because of the scenario structure, one of the first parameters to evaluate is the MT's transmission power as a function of the distance to the BS. From Fig. 2, it can be seen that all curves show an expected upwards trend in the required transmission power as the MT gets farther away from the BS and enters the repeater's area of influence. By comparing cases, one can observe that differences with respect to the full MRC approach reach up to $3 \mathrm{~dB}$ for the case of a repeater with 9 or $11 \mu$ s delay, because the path from the BS is out of the Rake time window $(20 \mu \mathrm{s})$. This implies two facts: first, it puts in evidence the presence of a term of self-

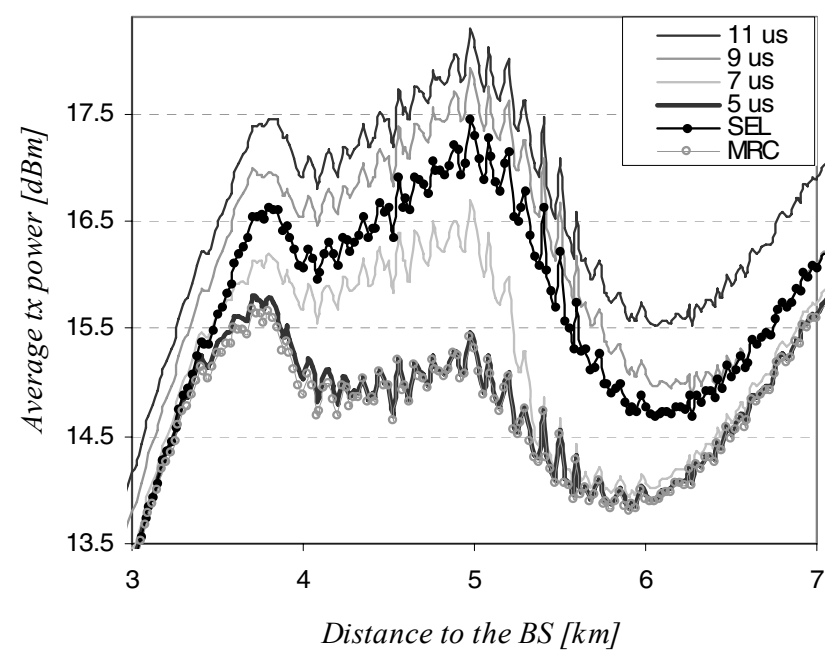

Fig. 2. UL transmission power as a function of the distance to the donor $\mathrm{BS}$, for classical approaches and different internal delays at the repeater.

interference that causes an increase of the transmission power; second, in this situation, fewer paths can be combined, and therefore the attenuation suffered by the MT is greater than the one measured in a full MRC case. The percentage of area in which all the paths cannot be constructively combined is directly proportional to the repeater internal delay, therefore, the degradation observed with respect to the too optimistic classical consideration will also increase. Notice that with an internal repeater delay of $5 \mu \mathrm{s}$, there are very few areas in which all the paths cannot be combined, hence, results are very similar to the full MRC case. For distances over $7 \mathrm{~km}$, we can show that the error obtained when using a classical approach keeps reducing until reaching a constant value of $1.5 \mathrm{~dB}$. Under some circumstances, inaccuracies of this order may not be tolerable.

On the other hand, if just the best path is considered (SEL case) and the repeater had an internal delay of $5 \mu \mathrm{s}$, an error around $2 \mathrm{~dB}$ occurs by the approximated analysis along $1 \mathrm{~km}$. The SEL curve tends to the MRC case when the MT is close to the BS. This behaviour is coherent, since the propagation path through the repeater is more than $10 \mathrm{~dB}$ below than that of the path through the BS, therefore, this signal contribution can be considered negligible. Conversely, when the MT reaches the repeater and continues moving away from the BS, the curve tends to the $9 \mu \mathrm{s}$ case. The curve does not tend to the $11 \mu$ s case because the SEL approximation only considers one path, the others not being taken into account, and then not generating any kind of interference. Between these two clear areas, the results are more or less accurate depending on the internal delay of the repeater and the actual possibility of some signal replicas to be out of the Rake window.

Considering that all MTs have a maximum available transmission power ( $21 \mathrm{dBm}$-Table I- in our scenario), the previous reasoning induces to think that there are also important differences in the prevision of the percentage of users not reaching their $E_{b} / N_{0}$ target (degraded users) between the classical approaches and the proposed analysis. Some results for this evaluation are shown in Table II, which actually shows the mean percentage of correctly served users. These values are 
TABLE II

EVOLUTION OF CORRECTLY SERVED USERS FOR ClASSICAL APPROACHES AND DIFFERENT INTERNAL DELAYS AT THE REPEATER

\begin{tabular}{|c|c|}
\hline Classical Approach / Internal Delay & Users [\%] \\
\hline \hline MRC & 95.3 \\
\hline SEL & 94.4 \\
\hline $5 \mu \mathrm{s}$ & 93.9 \\
\hline $7 \mu \mathrm{s}$ & 93.0 \\
\hline $9 \mu \mathrm{s}$ & 89.5 \\
\hline $11 \mu \mathrm{s}$ & 86.1 \\
\hline
\end{tabular}

obtained after analyzing all MTs at the end of each simulated snapshot. According to the full MRC or SEL approximations, around $95 \%$ of users can be correctly served, which is a typical design constraint. However, this value is reduced to $86 \%$ when the repeater has an internal delay of $11 \mu \mathrm{s}$. Therefore, the optimistic result may lead to accept as good an inappropriate system design when not using the proposed analysis. The differences are always higher with respect to the MRC approach, which is the most optimistic as it considers that all paths are always constructively combined. Even with a repeater with very low internal delay $(5 \mu \mathrm{s})$ a difference of $1.4 \%$ of users arises.

The curves on Fig. 3 compare the normalized histogram of the DL transmitted power per MT obtained from the simulated samples. Since the orthogonality factor exhibits significant temporal variations [18], two typical cases [19] of loss of orthogonality have been considered: in the first one, a coefficient (named 'rho' in the caption) equal to 0.6 is considered, and in the second one equal to 0.4 , which is more common in low dispersive channels. In this case, the most optimistic classical approach (MRC) is compared with the enhanced proposal considering a worst case commercial repeater with $11 \mu \mathrm{s}$ delay. It can be observed that the classical evaluation leads to a smoother error with respect to UL, since just a percentage of the total intra-cell power acts as interference; nevertheless, this error increases with the orthogonality factor. From the figure, it can be seen that for $\rho=0.6$ the histograms are $1 \mathrm{~dB}$ displaced, but the value is quite smaller in environments with $\rho=0.4$. The error is negligible as long as the accumulated delay in the link and repeater is under $5 \mu \mathrm{s}$, or if comparisons are drawn with the SEL case.

The same situations are subsequently evaluated in terms of coverage. Fig. 4 and Fig. 5 show the probability of coverage for each spatial point or pixel in the scenario. A pixel is considered to have coverage as long as it accomplishes these four conditions:

- The ratio of received energy per chip to the total power spectral density at the MT antenna connector $E_{c} / I_{0}$ measured on the pilot channel is higher than $-12 \mathrm{~dB}$. The pilot channel is realistically introduced according to UMTS features and so it allows MTs to execute cell selection and soft handover procedures [20] as well as estimate channel conditions.

- Once the MT has selected the cell, it has enough power so that the UL $E_{b} / N_{0}$ target is reached.

- Similarly, to reach the DL $E_{b} / N_{0}$ target, the MT does

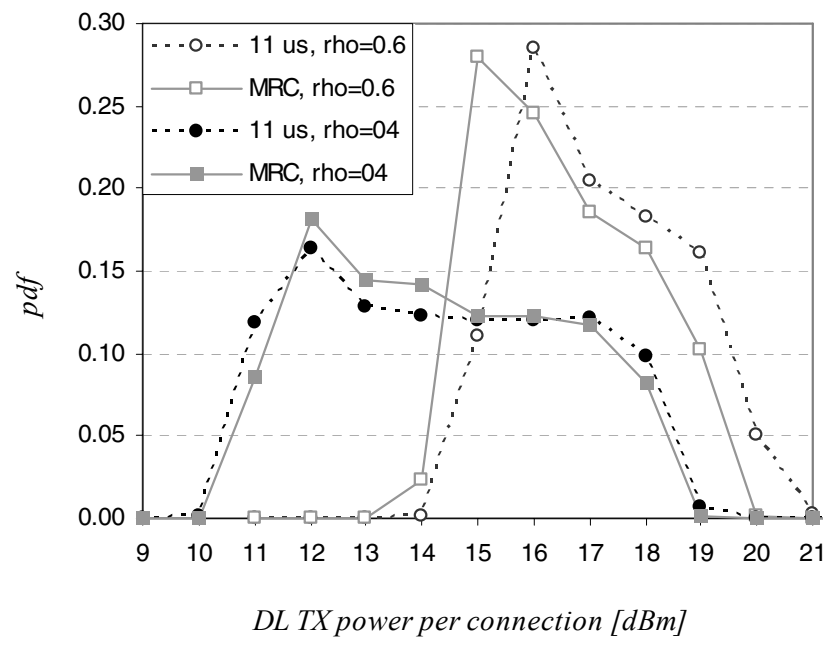

Fig. 3. DL transmission power of individual connections, comparing the $11 \mu$ s case with the classical full MRC approximation.

not need more power than the maximum allowed level per connection.

- The BS is not transmitting at its maximum transmission power, so it can serve new MTs.

Along the simulation and at the end of each snapshot, MTs and BS adjust their transmission powers. Under these load conditions, and before configuring a new sample, a test MT analyses all the pixels in the scenario and assesses the four conditions. This MT is transparent to the system and does not generate interference, i.e., it just acts as an evaluator and allows finding the probability of coverage for each pixel in the system realistically, without approximations.

It can be seen that, when the full MRC approach is used, with $\rho=0.4$ the coverage is supposed to be guaranteed in every point between the BS and the repeater. However, when considering the real delays of the paths, a clear loss is obtained, especially in the repeaters area of influence. With the proposed evaluation, certain zones turn out to demand more power than the available one and as a consequence connections are degraded or dropped. Since interference is also increased, the evaluated levels of $E_{c} / I_{0}$ on the pilot signals are affected too. These differences are much sharper when an orthogonality factor equal to 0.6 is considered, Fig. 5, in which case the analysis reveals that the proposed layout would be clearly inappropriate in a real situation. This conclusion would not be reached if a classical evaluation had been performed.

Note also that in Figs. 4 and 5, when our proposal is used, the coverage area of the repeater is not as regular as in the classical case; there is a central zone in which the coverage is slightly extended. Indeed, it corresponds to the portion of the scenario in which all paths fall inside the window of the Rake $\mathrm{RX}$ and the limit can be easily defined because the scenario is plain (it actually forms a hyperbola). Note that with classical approaches, these variations cannot be considered, since the coverage does not depend on the internal delay of the repeater or the link with the donor BS.

Finally, admission regions for the network have been studied. Admission Control (AC) is a key RRM strategy in WCDMA systems. Since coverage and capacity are tightly 


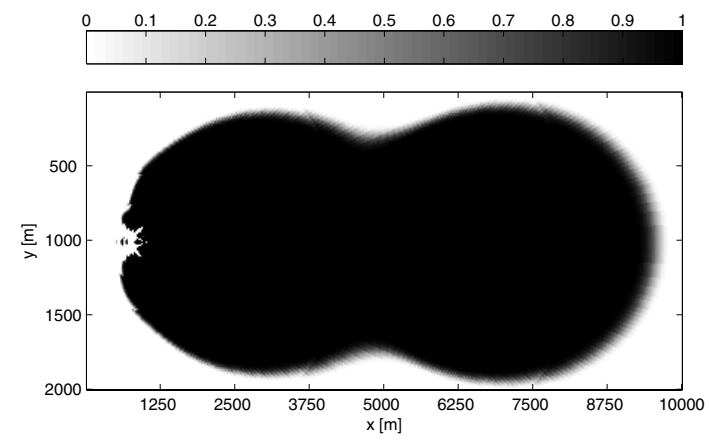

(a) Classical full MRC

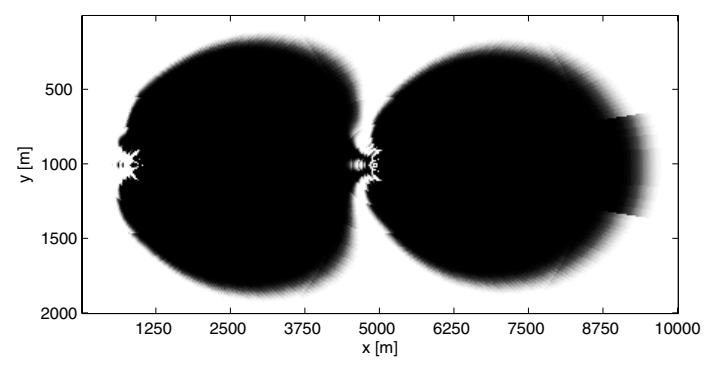

(b) Enhanced proposal

Fig. 4. Probability of coverage for internal delay $=11 \mu$ s and $\rho=0.4$.

coupled, a method that handles all new incoming traffic is mandatory. AC strategies decide whether a new radio access bearer can be admitted or not, according to a certain estimation of the current load. If the load stays below a certain threshold the new RAB will be allowed. The parameter $\eta(j)$ defined in (37) represents the load factor in the UL measured at BS $j$, being directly related to the noise rise due to interference [4]. In our simulations this maximum threshold has been adjusted to 0.85 , because it implies a typical value of $95 \%$ of users correctly served when evaluating the scenario with classical approaches.

$$
\eta(j)=\frac{\sum_{i=1}^{N_{M T}} P_{R X}(j, i)+n_{R X, R e p(j)}}{\sum_{i=1}^{N_{M T}} P_{R X}(j, i)+n_{B S}(j)+n_{R X, \operatorname{Rep}(j)}}
$$

Where:

- $P_{R X}(j, i)$ : Power received at BS $m$ from MT $i$.

In this sense, Fig. 6 shows the number of users that could be admitted in the study-case scenario. It can be seen that those results obtained when considering traditional approximations are optimistic. Indeed, when actual differences in delays are considered, the resulting admission region becomes smaller, being inversely proportional to the internal delay of the repeater. This degradation in capacity reaches the $8 \%$ when the repeater has an internal delay of $11 \mu \mathrm{s}$. Moreover, it can be seen that there is almost no difference between the MRC and SEL cases, because the only difference between these two approaches is the effective attenuation that perceives the MT; on the other hand, the enhanced also introduces selfinterference terms, which have a direct and evident impact on

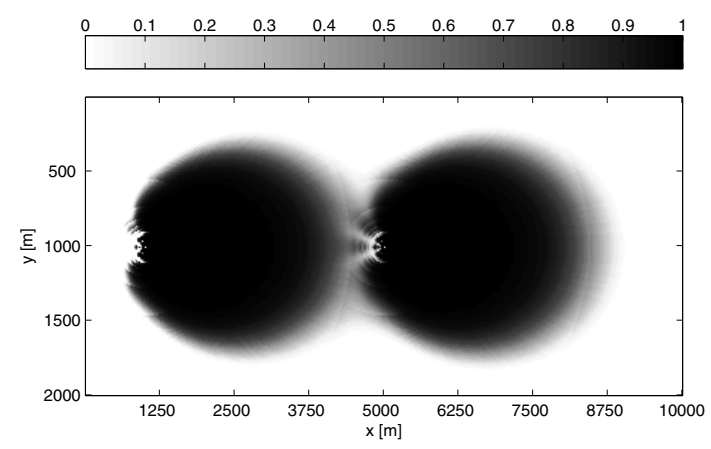

(a) Classical full MRC

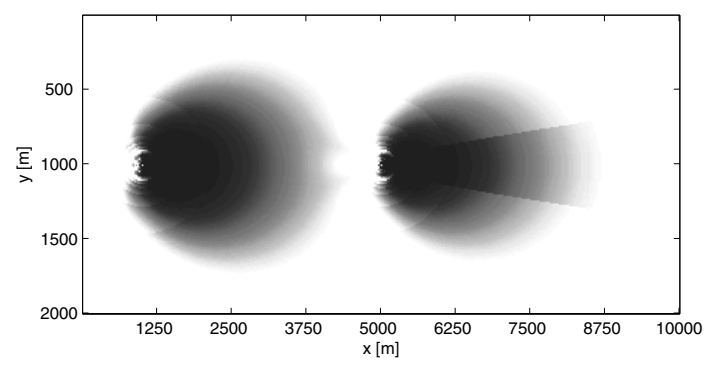

(b) Enhanced proposal

Fig. 5. Probability of coverage for internal delay $=11 \mu$ s and $\rho=0.6$.

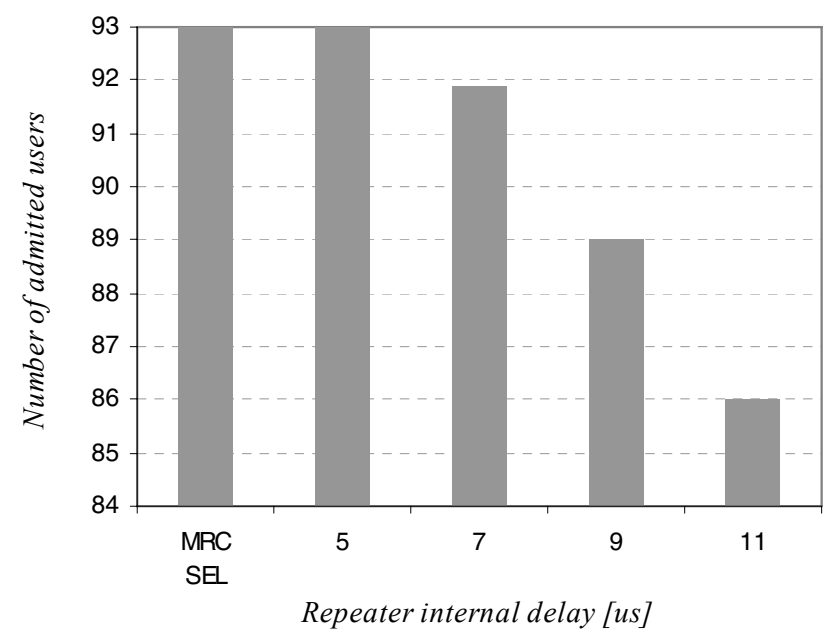

Fig. 6. Admission region evolution for classical approaches and different internal delays at the repeater.

the load factor (it is increased), and therefore, as it is shown, on the admission region size.

Thus, from the previous paragraphs it is clear that classical approaches tend to give biased and optimistic results, since they ignore important effects on the network performance indicators. The proposed analysis is concluded to be required for a proper system evaluation and planning.

\section{CONCLUSIONS}

Along this paper, a realistic analysis of WCDMA networks with repeaters deployment has been presented. Generic and compact expressions for UL and DL have been derived, so that transmitted powers can be calculated without simplifications 
and the effect of interference generated by signal replicas with such a delay that fall out of the Rake RX window has been considered realistically. In our mathematical approach, after defining different sets of mean propagation losses, effective equivalent losses have been introduced, allowing obtaining more compact and generic expressions. Similarly, for the same purpose, the SSIR in the UL and a derived term in the DL considering the $\rho$ factor have been introduced so that the problem has been able to be posed as an $N_{M T}$ th-order linear equations system. However, thanks to the relationships between $P_{T X}$ and $P_{T X, t o t}$ (for the UL and DL) a dimension reduction of the power control problem has been attained and has allowed its resolution with the same computational cost as in basic WCDMA systems. Moreover, solving the obtained equations systems is independent of the number of repeaters and, therefore, the proposed approach does not introduce new extra computational cost. The proposal considers realistically the paths delays and the finite duration of the time window in Rake RXs, which is why more accurate metrics can be obtained from a system level viewpoint.

Furthermore, with the help of numerical simulations, one has tested which are the major differences between the results obtained by the proposed analysis and the ones by the classical approaches. Different performance indicators of WCDMA networks have been studied, being shown that classical approximations lead to unrealistic and too optimistic results. This, for example, could lead to accept bad planned networks as good designs. UL and DL transmitted powers have been evaluated, UL being the most affected link in terms of transmitted power with differences up to $3 \mathrm{~dB}$. Because of the use of orthogonal codes, the differences in DL with respect to the classical approaches are smoothed, but especially higher accurate metrics are obtained in scenarios with high loss of orthogonality. The previsions on the number of non degraded mode users in the network would be also quite optimistic when classical approaches are used; in our example this value evolved from $95 \%$ to $86 \%$ when using the proposed method. Important reductions of coverage have been observed when the realistic behavior of the paths is considered. Finally, admission regions have been also compared, showing, one more time, that traditional approximations lead to inadequate too optimistic results. In the simulated layout, reductions in the number of admitted users can reach $8 \%$ when the analysis is done with the presented proposal.

Therefore, we conclude that the proposed analysis is a highly useful tool to plan and manage WCDMA networks that include the presence of repeaters.

\section{REFERENCES}

[1] M. Rahman and P. Ernstrom, "Repeaters for hotspot capacity in DSCDMA networks," IEEE Trans. Veh. Technol., vol. 53, no. 3, pp. 626633, May 2004

[2] E. Gago and L. Cucala, "Radio on fibre systems for practical $2 \mathrm{G}$ and 3G deployment," COST 273, Athens (Greece), Tech. Rep. available as TD(04)001, Jan. 2004.

[3] M. Garcia-Lozano, L. Alonso, F. Casadevall, and S. Ruiz, "Capacity and coverage tradeoff in WCDMA environments with repeaters deployment," Wireless Pers. Commun., vol. 40, no. 3, pp. 329-342, 2007.

[4] H. Holma and A. Toskala, WCDMA for UMTS Radio Access for Third Generation Mobile Communications, 2nd ed. Chichester, UK: John Wiley \& Sons, 2002.
[5] J. Zander, "Performance of optimum transmitter power control in cellular radio systems," IEEE Trans. Veh. Technol., vol. 41, no. 1, pp. 57-62, Feb. 1992.

[6] S. Jafar and A. Goldsmith, "Adaptive multirate CDMA for uplink throughput maximization," IEEE Trans. Wireless Commun., vol. 2, no. 2 , pp. 218-228, Mar. 2003.

[7] A. Baier, U. Fiebig, W. Granzow, W. Koch, P. Teder, and J. Thielecke, "Design study for a CDMA-based third-generation mobile radio system," IEEE J. Select. Areas Commun., vol. 12, no. 4, pp. 733-743, May 1994.

[8] W. Lee and D. Lee, "The impact of repeaters on CDMA system performance," in Proc. IEEE 51st Veh. Technol. Conf. (VTC 2000Spring), May 2000, pp. 1763-1767.

[9] M. Patwary, P. Rapajic, and I. Oppermann, "Capacity and coverage increase with repeaters in UMTS urban cellular mobile communication environment," IEEE Trans. Commun., vol. 53, no. 10, pp. 1620-1624, Oct. 2005.

[10] W. Choi, Y. Cho, and T. Ban, "Automatic on-off switching repeater for DS/CDMA reverse link capacity improvement," IEEE Commun. Lett., vol. 5, no. 4, pp. 138-141, Apr. 2001.

[11] K. Jeong, J. Cheong, T. Park, T. Kim, and S. Park, "Performance analysis of DS-CDMA reverse link with fiber-optics repeaters," in Proc. IEEE 51st Veh. Technol. Conf. (VTC 2000-Spring), May 2000, pp. 2439-2443.

[12] TR 25.956 (Release4) - UTRA Repeaters Planning Guidelines and System Analysis, 3GPP Specification [Online]. Available: http:// www. 3gpp.org/

[13] J. Laiho, A. Wacker, and T. Novosad, Radio Network Planning and Optimisation for UMTS, 2nd ed. Chichester, UK: John Wiley \& Sons, 2006.

[14] S. Handly, "Congestion measures in DS-CDMA networks," IEEE Trans. Commun., vol. 47, no. 3, pp. 426-437, Mar. 1999.

[15] L. Mendo and J. Hernando, "On dimension reduction for the power control problem," IEEE Trans. Commun., vol. 49, no. 2, pp. 243-248, Feb. 2001.

[16] E. Damoso and L. M. Correia, "COST 231 Final Report - Digital Mobile Radio: Evolution Towards Future Generation Systems," COST Secretariat, Brussels (Belgium), Tech. Rep., 1999.

[17] Kathrein website, 2006 [Online]. Available: http://www . kathrein.de/

[18] N. Mehta, L. Greenstein, T. Willis, and Z. Kostic, "Analysis and results for the orthogonality factor in WCDMA downlinks," IEEE Trans. Wireless Commun., vol. 2, no. 6, pp. 1138-1149, Nov. 2003.

[19] N. Mehta, A. Molisch, and L. Greenstein, "Orthogonality factor in WCDMA downlinks in urban macrocellular environments," in Proc. IEEE Global Telecommun. Conf. (GLOBECOM 2005), Nov./Dec. 2005, vol. 6.

[20] TS 25.133 (Release5) - Requirement for Support of Radio Resource Management (FDD), 3GPP Specification [Online]. Available: http: //www.3gpp.org/

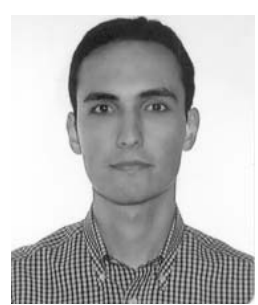

Mario Garcia-Lozano received the Degree in Telecommunications Engineering from the Technical University of Catalonia (UPC), Spain, in 2001. Currently, he is working toward the Ph.D. degree in Telecommunications Engineering. From 1999 to 2002 he was a member of the technical staff at Retevision, Spain, where he worked on the design and development of LMDS networks. In 2002 he joined the Department of Signal Theory and Communications at UPC, where he worked as a Research Assistant until 2003 under a grant from the Catalan Government. He currently lectures at the Castelldefels School of Technology (EPSC-UPC). He has actively participated in several research projects, funded by the European Union, the Spanish and Catalan Governments, and private companies. His research activities are focused in the field of mobile communication systems, especially radio network planning and radio resource management issues and optimization of cellular networks. 


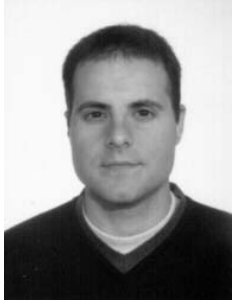

Luis Alonso (M'99) received the Engineer of Telecommunications degree from the Technical University of Catalonia (UPC), Spain, in 1997. In 1998 he joined the School of Telecommunications Engineering of Barcelona (ETSETB), Spain. He received the Ph.D. in Telecommunications Engineering from the Technical University of Catalonia (UPC) in 2001. The same year, he moved to the Castelldefels School of Technology (EPSC-UPC), Spain, in another campus within the same university, where he got a lecturer position while working in the Radio Communications Research Group of the Department of Signal Theory and Communications. In the beginning of 2006, he has reached a permanent tenured position in the university, becoming an Associate Professor (Profesor Titular). He has participated in several research programs, networks of excellence, COST actions, and integrated projects funded by the European Union and the Spanish Government, always working on the design and analysis of different mechanisms and techniques to improve wireless communications systems. He has also collaborated with some telecommunications companies as Telefónica, Alcatel, and Sener, working as a consultant for several research projects. He has also supervised a great number of masters theses, some of them developed by students working in companies such as Tradia, Orange, or Ericsson. He is currently the Project Coordinator of a Marie Curie Transfer of Knowledge Action (funded by the European Union) in collaboration with INA, a Greek Research Institute, and he is also the Scientific in Charge of a three-year research project funded by the Spanish Ministry of Science and Technology. His current research interests are within the fields of medium access protocols, radio resource management, cross-layer optimization, and QoS features for all kinds of wireless communications systems.

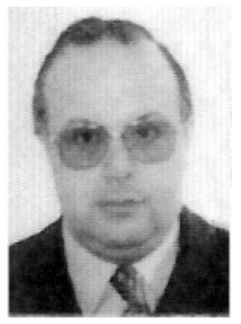

Fernando J. Casadevall (M'86) received the Engineer of Telecommunication and Dr. Engineering degrees from the Technical University of Catalonia (UPC), Spain, in 1977 and 1983 respectively. In 1978 he joined UPC, where he was an Associate Professor from 1983 to 1991 . He is currently Full Professor in the Signal Theory and Communications Department. After graduation he was concerned with equalisation techniques for digital fibre optic systems. He has also been working in the field of digital communications with particular emphasis on digital radio and its performance under multipath propagation conditions. In the last fifteen years, he has mainly been concerned with the performance analysis and development of digital mobile radio systems. In particular, his research interests include cellular and personal communication systems, multipath transceiver design (including Software Radio techniques), Mobility and Radio Resources Management, and End-to-End QoS issues. During the last ten years he participated in more than thirty research projects funded by both public and private organizations. In particular, he actively participated in ten research projects founded by the European Commission, being the Project Manager of three of them: ARROWS, EVEREST and AROMA. (See http://www.gcr.tsc.upc.edu for details.) Prof. Casadevall has published around one hundred technical papers in both international conferences and magazines; most of them correspond to IEEE publications. He has also been a Technical Program Committee member of different international IEEE supported conferences, as well as a reviewer of several IEEE magazines. From October 1992 to January 1996 he was responsible for the Information Technology Area in the National Agency for Evaluation and Forecasting (Spanish Nation Research Council).

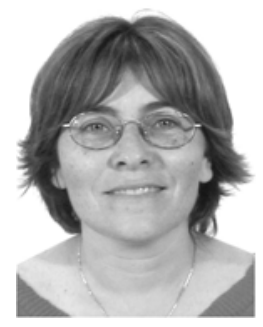

Silvia Ruiz (M'86) received the Engineer and Doctor Engineer degrees in telecommunication from the Technical University of Catalonia (UPC), Spain, in 1986 and 1989 respectively. She joined the Signal Theory and Communications Department, becoming Associate Professor in 1992. She has participated in several UE projects, COST actions, and Network of Excellence projects, as well as in projects funded by the Spanish government and private companies (Alcatel, Vodafone. Telefónica Móviles, etc.) She has also strongly collaborated in university management issues and has been Vice-Dean of Academic Affairs for three years, and Assistant Director of External Relations of the Castelldefels School of Technology (EPSC-UPC) since December 2005. Her research interests are in the field of mobile communication systems, especially radio network planning issues and optimization of $3 \mathrm{G}$ and heterogeneous networks.

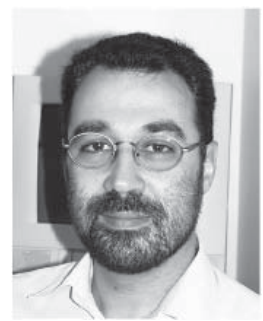

Luis M. Correia (S'85-M'91-SM'03) was born in Portimao, Portugal, in October 1958. He received the Ph.D. in Electrical and Computer Engineering from IST-TUL (Technical University of Lisbon) in 1991, where he is currently a Professor in Telecommunications, with his work focused in wireless/mobile communications in the areas of propagation, channel characterisation, radio networks, traffic, and services. He has acted as a consultant for Portuguese GSM operators and the telecommunications regulator. Besides being responsible for research projects at the national level, he has been active in various ones within European frameworks (RACE, ACTS, IST, and COST). He participated in and was co-editor of the Final Report for COST 231, and Chairman and Editor of the Final Reports of COST 259 and COST 273. He was and is responsible for the supervision of students at both the M.Sc. and Ph.D. levels, having authored many papers and communications in international journals and conferences, for which he has served also as a reviewer, editor, and board member. He has served as evaluator and auditor in ACTS, ESPRIT, and IST frameworks. He was the Chairman of the Technical Programme Committee of PIMRC'2002. $\mathrm{He}$ is part of the Expert Advisory Group and of the Steering Board of the European eMobility platform, and of the COST Domain Committee on ICT. 